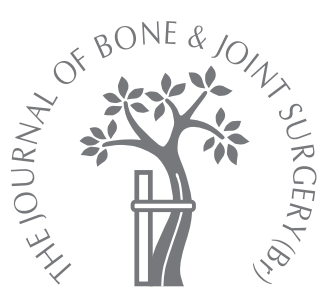
A. J. Butt,
T. McCarthy,
I. P. Kelly,
T. Glynn,
G. McCoy

From Kilcreen

Regional

Orthopaedic

Hospital, Kilkenny,

Ireland

\title{
Sciatic nerve palsy secondary to post- operative haematoma in primary total hip replacement
}

A. J. Butt, FRCS(Ed), Registrar in Orthopaedics - T. McCarthy, FRCSI,

Specialist Registrar in Orthopaedics

I. P. Kelly, FRCS, Consultant Orthopaedic Surgeon

T. Glynn, FRCS, Consultant Orthopaedic Surgeon

G. McCoy, FRCS,

Consultant Orthopaedic

Surgeon

Kilcreen Regional

Orthopaedic Hospital

Kilcreen, County Kilkenny,

Ireland.

Correspondence should be sent to Mr A. J. Butt; e-mail: ahsanjbutt@hotmail.com

(C)2005 British Editorial Society of Bone and Joint Surgery

doi:10.1302/0301-620X.87B11. $16736 \$ 2.00$

$J$ Bone Joint Surg [Br] 2005;87-B:1465-7.

Received 12 May 2005;

Accepted 14 June 2005

Sciatic nerve palsy is a recognised complication of primary total hip replacement. In our unit this complication was rare with an incidence of $<0.2 \%$ in the past ten years. We describe six cases of sciatic nerve palsy occurring in 355 consecutive primary total hip replacements (incidence $1.69 \%$ ). Each of these palsies was caused by post-operative haematoma in the region of the sciatic nerve.

Cases, which were recognised early and surgically-evacuated promptly, showed earlier and more complete recovery. Those patients for whom the diagnosis was delayed, and who were therefore managed expectantly, showed little or no recovery. Unexpected pain and significant swelling in the buttock, as well as signs of sciatic nerve irritation, suggest the presence of haematoma in the region of the sciatic nerve.

It is, therefore, of prime importance to be vigilant for the features of a sciatic nerve palsy in the early post-operative period as, when recognised and treated early, the injury to the sciatic nerve may be reversed.

Sciatic nerve palsy is a recognised complication of total hip replacement (THR). ${ }^{1-3}$ Its incidence varies widely; a review of 18 series between 1973 and 1997 found a mean incidence of $0.8 \%$ (0\% to $2.8 \%)^{2}$

The incidence in our institution before June 2000 was $<0.2 \%$. However, we now report six cases of sciatic nerve palsy which occurred in 355 consecutive primary THRs in a one-year period representing an incidence of $1.69 \%$. All were associated with a haematoma in the region of the sciatic nerve. Our aim is to report the frequency of this complication and highlight the need for clinical awareness in the early post-operative period because, if recognised and treated early, the condition may be reversible.

\section{Patients and Methods}

We undertook a clinical review, with a minimum of three years' follow-up, of six patients who had developed a sciatic nerve palsy after THR. The following were recorded from the medical records: pre-operative details including medical history, body-weight, medication (aspirin, non-steroidal anti-inflammatory drugs, anticoagulants), bleeding disorders, haemoglobin level, international normalised ratio and activated partial thromboplastin time (Table I). Intra-operative details included type of anaesthesia, surgical approach and duration of sur- gery (Table II). Post-operative details included the use of drains, total blood loss, haemoglobin level, transfusion requirements, type of anticoagulant used and the time of presentation of the palsy after surgery (Table III). The signs, symptoms, management and outcome were recorded.

\section{Results}

The six patients were admitted for an elective, primary THR. None had a history of a bleeding disorder. Pre-operatively, one patient was taking aspirin and one was taking nimesalide. All pre-operative haemoglobin levels and coagulation profiles were within normal limits. Five had a body-weight of less than $70 \mathrm{~kg}$.

Surgery was performed through a posterior approach in all patients, the sciatic nerve being identified in all cases. Suction drains were used in four patients; the estimated total blood loss was less than expected from the significant falls in haemoglobin levels (Table III), suggesting an occult blood loss. Five patients received tinazaparin sodium (4500 IU subcutaneously once daily) and one enoxaparin sodium (40 mg subcutaneously once daily). All received anticoagulation within the first six post-operative hours.

None had significant symptoms after the effects of the spinal anaesthesia had disappeared, and all were documented to have nor- 
Table I. Pre-operative findings

\begin{tabular}{lllllll}
\hline Case & $\begin{array}{l}\text { Bleeding } \\
\text { disorder }\end{array}$ & NSAIDs $^{*}$ & $\mathbf{H b}^{\dagger} \mathbf{( g / d l )}$ & INR $^{\ddagger}$ & APTT $^{\S}$ & $\begin{array}{l}\text { Body weight } \\
\mathbf{( k g )}\end{array}$ \\
\hline $1^{\Uparrow}$ & No & No & 12.3 & 1.0 & 31 & 66 \\
2 & No & Aspirin & 12.4 & 0.9 & 28 & 43 \\
3 & No & No & 14.5 & 1.0 & 29 & 66 \\
4 & No & Nimesulide & 14.5 & 0.9 & 27 & 58 \\
5 & No & No & 14.4 & 1.0 & 29 & 86 \\
6 & No & No & 10.4 & 1.0 & 28 & 60 \\
\hline
\end{tabular}

* NSAIDs, non-steroidal anti-inflammatory drugs

$\dagger \mathrm{Hb}$, haemoglobin level

‡ INR, international normalised ratio

$\S$ APTT, activated partial thromboplastin time

I had a history of diabetes mellitus

Table II. Peri-operative findings

\begin{tabular}{lllcl}
\hline Case & $\begin{array}{l}\text { Surgical } \\
\text { approach }\end{array}$ & $\begin{array}{l}\text { Type of } \\
\text { anaesthesia }\end{array}$ & $\begin{array}{l}\text { Operative } \\
\text { duration (mins) }\end{array}$ & Drains \\
\hline 1 & Posterior & Spinal & 75 & No \\
2 & Posterior & Spinal & 90 & Yes \\
3 & Posterior & Spinal & 80 & No \\
4 & Posterior & Spinal & 100 & Yes \\
5 & Posterior & Spinal & 65 & Yes \\
6 & Posterior & Spinal & 75 & Yes \\
\hline
\end{tabular}

Table III. Post-operative findings

\begin{tabular}{llllll}
\hline Case & $\begin{array}{l}\text { Total blood } \\
\text { loss }(\mathbf{m l})\end{array}$ & $\mathbf{H b}^{*}$ (g/dl) & $\begin{array}{l}\text { Blood transfusion } \\
\text { (units) }\end{array}$ & $\begin{array}{l}\text { Presentation of } \\
\text { symptoms }(\mathbf{h r s} \\
\text { after surgery) }\end{array}$ & $\begin{array}{l}\text { Anticoagulant } \\
\text { used }\end{array}$ \\
\hline 1 & 130 & 7.5 & Nil & $\leq 48$ & Tinazaparin \\
2 & 750 & 6.7 & 9 & $\leq 48$ & Tinazaparin \\
3 & 105 & 7.7 & 7 & $\leq 48$ & Tinazaparin \\
4 & 946 & 8.1 & 2 & $\leq 48$ & Tinazaparin \\
5 & 580 & 9.9 & Nil & $\leq 24$ & Tinazaparin \\
6 & 1174 & $6.87^{\dagger}$ & 4 & $\leq 48$ & Enoxaparin \\
\hline
\end{tabular}

* Hb, haemoglobin level on the first post-operative day

$\dagger$ after transfusion of two units of red blood cells

mal active ankle movements. Later, however, they developed pain and swelling in the buttock, the pain radiating in the distribution of the sciatic nerve. It was graded as severe in all cases and required repeated analgesia. There was associated altered sensation in the distribution of the sciatic nerve and all developed progressive loss of dorsiflexion of the ankle and, within 48 hours, five had developed a complete drop foot. None of the six patients had any change in leg length after THR.

For the first three patients, the diagnosis was delayed and they were managed expectantly. Two of these made a slow and incomplete recovery by 55 and 52 months after surgery. The third patient had a complete drop foot 49 months after surgery.

For the remaining three patients, the diagnosis was made early because of our previous experience and increased awareness and the wound explored under general anaesthesia within 48 hours of the operation. In each of these cases, a significant haematoma ( 800 to $1000 \mathrm{ml}$ ) was found deep to the fascia lata, in the region of the sciatic nerve. No discrete bleeding point was identified and there was no evi- dence of iatrogenic damage to the nerve. These patients made an uneventful recovery, with immediate resolution of symptoms and recovery of sciatic nerve function within a period of two hours, 24 hours and two weeks, respectively. At the latest follow-up, it was more than 24 months after surgery, and there remained no adverse effects or neurological deficit in these patients.

\section{Discussion}

Sciatic nerve palsy is a recognised complication of THR, which occurs predominantly following the use of the transtrochanteric and posterior approaches. ${ }^{1,2}$ Neurological deficit is evident in the immediate post-operative period and may be caused by a direct injury, or traction on the sciatic nerve during surgery. ${ }^{2}$ Although rare, delayed sciatic nerve palsy after THR has been reported. ${ }^{3}$ Possible causes of sciatic nerve injury after THR include ${ }^{4,5}$ mechanical damage at the time of surgery, thermal injury by bone cement, mechanical damage after surgery, such as fraying over a cement osteophyte ${ }^{6}$ and injury because of traction from overlengthening the leg. ${ }^{7}$ 
We have reported six cases of sciatic nerve palsy in 355 consecutive primary total hip replacements. In each case, there was sciatic nerve function when the effects of the spinal anaesthesia had worn off and the nerve palsy only developed over the subsequent 24 to 48 hours. All patients reported paraesthesiae in the ipsilateral foot and had marked swelling in the region of the sciatic nerve. A clinical picture of complete drop foot developed in five patients; the last case was identified before the loss of power was total. Each patient had a significant drop in their haemoglobin level, which could not be solely accounted for by the operative and post-operative blood losses, and suggested occult bleeding.

We also noted that five patients weighed less than the mean of $70 \mathrm{~kg}$ but still received a full, prophylactic dose of anticoagulant. We suspect that this may explain why they developed a haematoma, unless they were more sensitive to the anticoagulant used for prophylaxis. Because of this, we changed our practice by administering a reduced dose of anticoagulant to patients who weighed less than $70 \mathrm{~kg}$.

In the first three cases, because the diagnosis was not made early the patients were treated expectantly and an ankle-foot orthosis was used for the drop foot.

In the second three cases, each patient was taken back to the operating theatre within 48 hours of their THR for wound exploration under general anaesthesia, a significant haematoma being evacuated from the region of the sciatic nerve. Interestingly, the last of these six patients was in the process of developing the signs of a sciatic nerve palsy, in the form of increasing pain, paraesthesiae and gradual loss of dorsiflexion, but had not developed a complete drop foot. Because of our increased awareness, this patient was diagnosed and treated promptly, with an immediate reversal of all her symptoms. Each of these patients had an uneventful post-operative period, with complete symptomatic recovery before discharge from hospital. We did not have easy access to nerve conduction studies in our unit, so the exact neurological injury could not be documented.

We conclude that sciatic nerve palsy after primary THR is a significant and serious complication. It can be caused by pressure from a haematoma in the region of the sciatic nerve, especially in patients of less than $70 \mathrm{~kg}$ body-weight who have received a full dose of prophylactic anticoagulant, or in patients who are more sensitive to the anticoagulant used. We also conclude that drains do not always prevent haematoma formation and recommend increased vigilance in the early post-operative period after a primary THR. Patients complaining of an unexpected degree of pain in the gluteal region may be developing sciatic nerve irritation. We strongly advocate prompt evacuation of the haematoma once a clinical diagnosis of sciatic nerve compression has been made. Early damage to the sciatic nerve may be reversed.

No benefits in any form have been received or will be received from a commercial party related directly or indirectly to the subject of this article.

\section{References}

1. Ratliff AHC. Vascular and neurological complications. In: Ling RSM, ed. Complications of total hip replacement. London: Churchill-Livingstone, 1984:23-6.

2. Mason WTM, Burgess BM, Villar RN. A little too close to the bone?: risk of sciatic nerve injury during hip arthroplasty. Hip Int 2000;10:166-9.

3. Katsimihas M, Hutchinson J, Heath P, Smith E, Travlos J. Delayed transient sciatic nerve palsy after total hip replacement. J Arthroplasty 2002;17:203-7.

4. Fleming RE Jr, Michelsen CB, Stinchfield PE. Sciatic paralysis: a complication of bleeding following hip surgery. J Bone Joint Surg [Am]1979;61-A:37-9.

5. Nadeem RD, Clift BA, Martindale JP. Acute compartment syndrome of the thigh after joint replacement with anticoagulation. J Bone Joint Surg [Br] 1998;80-B:866-8.

6. Oleksak M, Edge AJ. Compression of the sciatic nerve by methylmethacrylate cement after total hip replacement. J Bone Joint Surg [Br] 1992;74-B:729-30.

7. Johnson NA, Pellicci PM, Tsairis P, Salvati EA. Nerve injury in total hip arthroplasty. Clin Orthop 1983;179:214-22. 